\title{
The Effects of the VFSS Timing After Nasogastric Tube Removal on Swallowing Function of the Patients With Dysphagia
}

\author{
Du Hyeon Nam, MD, A Young Jung, MD, Ji Hwan Cheon, MD, \\ Howard Kim, Eun Young Kang, MD, Sung Hoon Lee, MD
}

Department of Physical Medicine and Rehabilitation, Kwangju Christian Hospital, Gwangju, Korea

\begin{abstract}
Objective To evaluate the effects of the videofluoroscopic swallowing study (VFSS) timing after the nasogastric tube (NGT) removal on swallowing function of the patients with dysphagia.

Methods This study was conducted on 40 NGT-fed patients with dysphagia. To assess the patients' swallowing function, VFSS was performed twice using a 5-mL $35 \%$ diluted barium solution. For the initial examination, VFSS was performed immediately after the NGT removal (VFSS 1). For the second examination, VFSS was performed five hours after the NGT removal (VFSS 2). We used the functional dysphagia scale (FDS) to assess swallowing function. In the FDS, a significant difference in the four items in the oral phase, seven items in the pharyngeal phase, and total scores were assessed $(\mathrm{p}<0.05)$. We also used modified penetration-aspiration scale (mPAS) to compare the two examinations $(\mathrm{p}<0.05)$.

Results A paired t-test was performed to confirm the statistical significance of the two examinations $(\mathrm{p}<0.05)$. The overall swallowing function was assessed as better in VFSS 2 than in VFSS 1 . In the FDS, significant differences in the residue in valleculae $(\mathrm{p}=0.002)$, the residue in pyriform sinuses $(\mathrm{p}=0.001)$, the coating of pharyngeal wall after swallow $(\mathrm{p}=0.001)$, and the total scores $(\mathrm{p}<0.001)$ were found between the two examinations. Also, in the mPAS that assessed the degree of penetration-aspiration, a significant difference was found between the two examinations $(\mathrm{p}<0.001)$.

Conclusion The results of this study confirmed that the timing of the VFSS after the NGT removal affects the swallowing function. Thus, to accurately assess the swallowing function, VFSS must be performed in NGT-fed patients after they have rested for a certain period following the removal of their NGT.
\end{abstract}

Keywords Dysphagia, Enteral Nutrition, Deglutition

Received October 10, 2014; Accepted January 23, 2015

Corresponding author: Sung Hoon Lee

Department of Physical Medicine and Rehabilitation, Kwangju Christian Hospital, 37 Yangnim-ro, Nam-gu, Gwangju 503-715, Korea

Tel: +82-62-650-5162, Fax: +82-62-671-7447, E-mail: starhoon3@hanmail.net

(ㄷ) This is an open-access article distributed under the terms of the Creative Commons Attribution Non-Commercial License (http://creativecommons.org/ licenses/by-nc/4.0) which permits unrestricted noncommercial use, distribution, and reproduction in any medium, provided the original work is properly cited. Copyright $\odot 2015$ by Korean Academy of Rehabilitation Medicine 


\section{INTRODUCTION}

Dysphagia, which accompanies various diseases, including stroke, is an important clinical problem since it causes serious sequelae such as aspiration pneumonia and malnutrition, and reduces the quality of life $[1,2]$. Various examinations have been developed and used to evaluate accurately the swallowing function, which is important in determining the treatment direction for dysphagia patients [3]. Among these examinations, videofluoroscopic swallowing study (VFSS) has been used as the gold standard assessing dysphagia patients $[4,5]$.

Nasogastric tube (NGT) is easy to apply and relatively less invasive. Thus, it is commonly used to provide adequate nutrition to dysphagia patients. For this reason, it is applied to the majority of dysphagia patients who require VFSS. However, as the VFSS protocol does not include the criteria for the timing of the NGT removal for NGT-fed patients, the timing of the NGT removal varies depending on the physician.

Unfortunately, prior to this study, no study had been conducted on the effect of the timing of the NGT removal on swallowing function. Accordingly, this study was conducted to investigate the effect of the VFSS timing after the NGT removal on VFSS outcomes.

\section{MATERIALS AND METHODS}

\section{Subjects}

Of the patients hospitalized at Kwangju Christian Hospital from October 2013 to August 2014, 52 who had NGT due to dysphagia were included in this study. Twelve patients were withdrawn due to the exclusion criteria, and the study was prospectively conducted on the remaining 40 patients. The patients who met the following criteria were candidates for this study: 1) had NGT due to dysphagia, and partial or complete nutrition via the NGT before the VFSS; 2) had no systemic infection at the time of the VFSS; and 3) understood the study purpose and agreed to participate in the study.

The mean age of the patients was 63.3 years (range, 15-81 years), and 21 of them were male and 19 were female. Of the 24 patients who had stroke, eight had ischemic stroke and 16 had hemorrhagic stroke. In addition, 10 had traumatic brain injury; five, respiratory diseases such as chronic obstructive pulmonary disease (COPD); and one, hypoxic brain injury. All the patients used 18 French-sized NGTs, and the mean period of their NGT use was 106 days (range, 6-882 days). The patients' other characteristics are presented in Table 1.

The exclusion criteria were as follows: 1) difficulty in coordination, or difficulty in maintaining a seated position; 2) difficulty in interpreting the results; 3) severe aspiration that might threaten the patient's safety (e.g., a modified penetration-aspiration scale [MPAS] is 5); and 4) a significant change in the patient's condition between the two examinations.

This study was approved by the Ethics Committee of the Kwangju Christian Hospital, and written informed consent of the patients was obtained.

\section{Study protocol}

VFSS was performed by a doctor from the Department of Rehabilitation, with the help of occupational therapists and radiologic technicians. It was performed twice (in the morning and in the afternoon) in NGT-fed patients with dysphagia. For the initial examination, the patients were asked to visit the examination room at 9:00 AM. with the NGT inserted, and then the VFSS was conducted immediately after the NGT had been removed. After the patients were asked to sit down laterally to secure their anatomical structure, they were asked to swallow $5 \mathrm{~mL}$ of

Table 1. Demographics and clinical characteristics of subjects $(n=40)$

\begin{tabular}{lc}
\hline \multicolumn{1}{c}{ Demographic factor } & Value \\
\hline Age (yr) & $63.30 \pm 16.95$ \\
Sex (male:female) & $21: 19$ \\
Preceding disease & \\
\hline Stroke (ischemic:hemorrhagic) & $24(8: 16)$ \\
Traumatic brain injury & 10 \\
\hline Pulmonary disease (COPD) & 5 \\
Hypoxic brain injury & 1 \\
Period of nasogastric tube insertion (day) & 106 \\
Number of dysphagia treatment & 7.38 \\
K-MMSE & 12.25 \\
Endotracheal tube & 8 \\
\hline
\end{tabular}

Values are presented as mean or mean \pm standard deviation.

COPD, chronic obstructive pulmonary disease; K-MMSE, Korean version of Mini-Mental State Examination. 
a 35\% diluted barium solution twice using a syringe (VFSS 1). For the second examination, after the NGT had been removed, the patients were kept in that condition for five hours. Then VFSS was performed at 2:00 PM in the same manner as VFSS 1 (VFSS 2).

\section{Interpretation}

The VFSS images were analyzed by two rehabilitation specialists with at least six years of experience in VFSS interpretation. We used the functional dysphagia scale (FDS) (Appendix 1) to assess the overall swallowing function. The FDS was developed by Han et al. [6], and the clinical eligibility was proven by validating its close association with the American Speech-LanguageHearing Association National Outcomes Measurement System (ASHA-NOMS) swallowing level scale, the relative weighing was applied to the physiological scale, which is closely associated with aspiration, and the scale, which consists of four items in the oral phase and seven items in the pharyngeal phase, was assessed [6-8]. We also used the mPAS (Appendix 2) to determine penetration-aspiration degree. The mPAS was revised from the penetrationaspiration scale [9] based on penetration, aspiration followed by cough reflex, and aspiration without cough reflex [10]. In the mPAS, 1-5 scales were converted into 1-5 points, respectively, and then compared between the two examinations $(\mathrm{p}<0.05)$.

\section{Statistical analysis}

A statistical analysis was performed using SPSS ver. 18.0 (SPSS Inc., Chicago, IL, USA). A paired t-test was conducted to confirm the significant difference between the VFSS 1 and VFSS 2 results $(\mathrm{p}<0.05)$.

\section{RESULTS}

In the FDS, no significant difference was found between the two examinations for the items in the oral phase. However, for the items in the pharyngeal phase, significant differences in the residue in valleculae $(\mathrm{p}=0.002)$, the residue in pyriform sinuses $(\mathrm{p}=0.001)$, and the coating of pharyngeal wall after swallow $(p=0.001)$ were found between the two examinations. In addition, a significant difference was found between the total scores in the two examinations $(p<0.001)$, which were obtained from the summation of the results of the oral phase and the pharyngeal phase (Table 2 ).

As for the mPAS, aspiration (scale 4) was observed in nine patients in VFSS 1, but only in four patients in VFSS 2. Penetration (scale 2 and 3 ) was observed in 22 patients in VFSS 1 , but in only 17 patients in VFSS 2 . Scale 1 , which shows neither aspiration nor penetration, was observed

Table 2. Differences in the results of the FDS between VFSS 1 and VFSS $2(n=40)$

\begin{tabular}{lccc}
\hline & VFSS 1 & VFSS 2 & p-value \\
\hline Total score & $33.96 \pm 16.20$ & $26.76 \pm 15.00$ & $<0.001^{*}$ \\
\hline Oral phase & & & 0.32 \\
\hline Lip closure & $0.38 \pm 1.33$ & $0.25 \pm 1.10$ & 0.08 \\
\hline Bolus formation & $1.20 \pm 1.78$ & $0.98 \pm 1.42$ & 0.58 \\
\hline Residue in oral cavity & $0.85 \pm 1.27$ & $0.60 \pm 1.03$ & 0.08 \\
\hline Oral transit time & $1.95 \pm 2.85$ & $1.50 \pm 2.63$ & \\
Pharyngeal phase & & & 0.16 \\
\hline Trigger of pharyngeal swallow & $5.00 \pm 5.03$ & $4.50 \pm 5.04$ & 0.08 \\
\hline Laryngeal elevation and epiglottic closure & $10.20 \pm 4.34$ & $9.30 \pm 5.08$ & 0.32 \\
\hline Nasal penetration & $0.20 \pm 0.88$ & $0.10 \pm 0.63$ & $0.002^{*}$ \\
\hline Residue in valleculae & $3.80 \pm 2.39$ & $2.90 \pm 2.22$ & $0.001^{*}$ \\
\hline Residue in pyriform sinuses & $3.60 \pm 3.11$ & $2.60 \pm 2.65$ & $0.001^{*}$ \\
\hline Coating of pharyngeal wall after swallow & $4.50 \pm 5.04$ & $2.00 \pm 2.02$ & 0.16 \\
\hline Pharyngeal transit time & $2.30 \pm 2.00$ & $2.10 \pm 2.02$ & \\
\hline
\end{tabular}

Values are presented as mean \pm standard deviation.

VFSS, videofluoroscopic swallowing study; FDS, functional dysphagia scale.

${ }^{*} \mathrm{p}<0.05$. 
Table 3. The results of the mPAS between VFSS 1 and VFSS $2(\mathrm{n}=40)$

\begin{tabular}{ccc}
\hline Scale & VFSS 1 & VFSS 2 \\
\hline 1 & 9 & 19 \\
2 & 14 & 13 \\
3 & 8 & 4 \\
4 & 9 & 4 \\
5 & 0 & 0 \\
\hline
\end{tabular}

VFSS, videofluoroscopic swallowing study; mPAS, modified penetration-aspiration scale.

Table 4. Differences of the results of the mPAS between VFSS 1 and VFSS $2(n=40)$

\begin{tabular}{cccc}
\hline & VFSS 1 & VFSS 2 & p-value \\
mPAS & $2.40 \pm 1.06$ & $1.83 \pm 0.98$ & $<0.001^{*}$ \\
\hline
\end{tabular}

Values are presented as mean \pm standard deviation. VFSS, videofluoroscopic swallowing study; mPAS, modified penetration-aspiration scale.

${ }^{*} \mathrm{p}<0.05$.

in nine patients in VFSS 1 , but in 19 patients in VFSS 2 (Table 3). In the comparison of the mPASs between the two examinations, a significant difference in the penetration-aspiration degrees was found $(p<0.001)$ (Table 4 , Fig. 1). There have been no scale changes in 22 patients, one scale decline in 13 patients, and two scale decline in 5 patients, whereas no patients showed scale risen in VFSS 2 compared with VFSS 1.

\section{DISCUSSION}

In the FDS, no significant difference was found between the two examinations for the four items in the oral phase. This is likely to have resulted from the NGT having nasally inserted and passed by the nasopharynx and esophagus, without passing by the oral cavity.

For the items in the pharyngeal phase, significant differences in the residue in valleculae, the residue in pyriform sinuses, and the coating of pharyngeal wall after swallow were found, but no significant difference in the other four items was found. The following hypothesis is made to explain the aforementioned result. First, there is a sensory problem such as desensitization in the pharyngeal structures, as NGT insertion for more than a certain period could impose a constant foreign body sensation on the pharyngeal structures, which would desensitize the

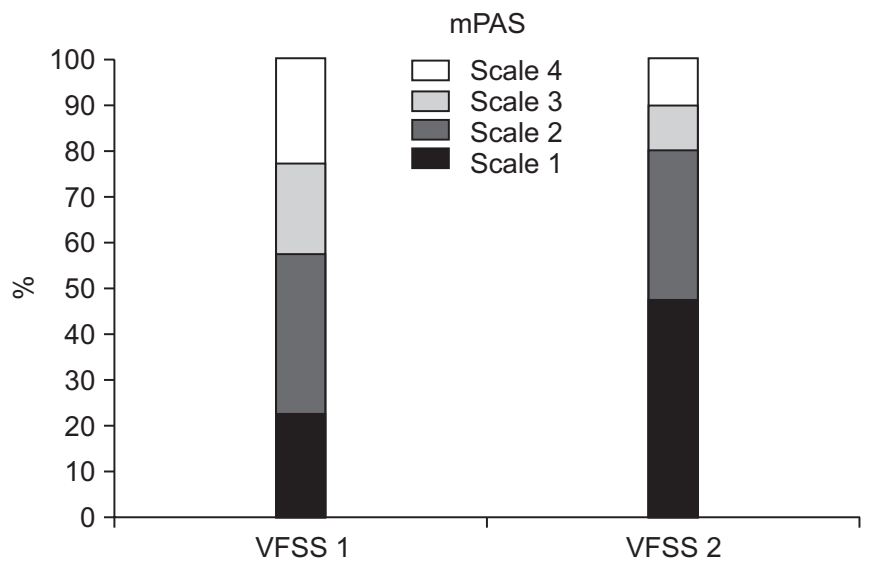

Fig. 1. The result of the modified penetration-aspiration scale (mPAS) between VFSS 1 and VFSS $2(n=40)$. VFSS, videofluoroscopic swallowing study.

sensory organs. Such desensitization could last for a long time even after the NGT removal. Food intake in such a condition could reduce the sensory ability of the desensitized pharynx. This could in turn delay the response time of the pharyngeal muscle or reduce the response intensity, which would produce valleculae or pyriform sinus residues. Then, the swallowing response was likely to have been improved in VFSS 2 due to the reduction of the desensitization through the five-hour stabilization after the NGT removal. Wang et al. [11] reported that no return of the desensitized pharyngeal wall to the normal state was observed 30 minutes after the NGT removal. Second, there could have been a dyscoordination between the swallowing-related muscles. NGT insertion may affect various swallowing-related muscles, such as the tongue base or the epiglottis, which has intermittent contact with the NGT, and the cricopharyngeus muscle, which has constant contact with the NGT. Delicate coordination between these structures is required within a short time for normal swallowing $[12,13]$. However, if the effect on each structure varies during the NGT insertion, dyscoordination may occur. In this study, dyscoordination was less observed in VFSS 2 than in VFSS 1 . In addition, a relatively lower volume of valleculae and pyriform sinus residues and a lower penetration-aspiration degree were observed in well-coordinated patients. These are likely to have been due to the improved swallowing on account of the reduction of the dyscoordination of the pharynx muscles through the five-hour stabilization after the NGT removal.

A significant difference between the mPASs in the two 
examinations was found. The valleculae and pyriform sinus residues may act as risk factors of aspiration. If this state is accompanied by dyscoordination, it further increases the aspiration, which would eventually increase the penetration-aspiration degree.

Long-term NGT insertion may cause various complications, such as lesions in the nasal wing, chronic sinusitis, gastro-esophageal reflux, and aspiration pneumonia [14]. Thus, if no evidence of aspiration is found in VFSS, quicker removal of the NGT is recommended. However, if the NGT is removed just before VFSS in NGT-fed patients, the patients' swallowing function may be underestimated, as shown in this study. If such situation occurs, it delays oral diet who has a good swallowing function which would increase the NGT-induced complications and delay the patients' early rehabilitation. Thus, resting for a certain period after the NGT removal would be helpful in accurately assessing the swallowing functions and plays an important role in determining whether to replace the NGT feeding with oral feeding.

This study had several limitations. First, on the subject selection, patients who had a certain degree of swallowing function and those with severe aspiration were excluded for their safety. Accordingly, there could have been a selection bias, as severe dysphagia patients were excluded. Second, the effect of the five-hour-fasting state on swallowing function and the test compliance was not ruled out. VFSS 1 was performed after breakfast, whereas VFSS 2 was performed before lunch. Actually, it was difficult to perform VFSS in patients with cognitive impairment due to their satiety after a meal. This could make the interpretation of the results difficult. Third, the most ideal timing of the NGT removal for VFSS was not presented. Thus, a further study is required to determine the ideal VFSS timing after the NGT removal, and this is expected to improve the accuracy and reliability of the assessment of swallowing function via VFSS.

In conclusion, this study showed that the VFSS timing after the NGT removal affected the FDS and mPAS by influencing the swallowing function. A significant difference in the residue in valleculae, the residue in pyriform sinuses, and the coating of pharyngeal wall after swallow were found between the two examinations, which also significantly affected the total FDS scores. In addition, a significant difference in the mPAS was found between the two examinations, which indicated that the VFSS tim- ing after the NGT removal also affected the penetrationaspiration degree. Therefore, to accurately assess the swallowing function, VFSS must be performed in NGTfed patients after a certain period of rest following the removal of their NGT.

\section{CONFLICT OF INTEREST}

No potential conflict of interest relevant to this article was reported.

\section{REFERENCES}

1. Smithard DG, O'Neill PA, Parks C, Morris J. Complications and outcome after acute stroke. Does dysphagia matter? Stroke 1996;27:1200-4.

2. Finestone HM, Greene-Finestone LS, Wilson ES, Teasell RW. Malnutrition in stroke patients on the rehabilitation service and at follow-up: prevalence and predictors. Arch Phys Med Rehabil 1995;76:310-6.

3. Palmer JB, Pelletier CA, Matsuo K. Rehabilitation of patients with swallowing disorders. In: Braddom RL, editor. Physical medicine and rehabilitation. 4th ed. Philadelphia: Saunders; 2011. p. 581-97.

4. Jung SH, Lee KJ, Hong JB, Han TR. Validation of clinical dysphagia scale: based on videofluoroscopic swallowing study. J Korean Acad Rehabil Med 2005;29:34350.

5. Eyal H. Evaluation and treatment of swallowing disorders (dysphagia): the American model as an opportunity for improving patient care and cost containment in Israel's healthcare system. Harefuah 1997;132:775-8.

6. Han TR, Paik NJ, Park JW. Quantifying swallowing function after stroke: a functional dysphagia scale based on videofluoroscopic studies. Arch Phys Med Rehabil 2001;82:677-82.

7. American Speech-Language-Hearing Association; National Outcomes Measurement System. Adult speech language pathology training manual. Rockville: American Speech-Language-Hearing Association; 1998.

8. Paik NJ, Kim IS, Kim JH, Oh BM, Han TR. Clinical validity of the functional dysphagia scale based on videofluoroscopic swallowing study. J Korean Acad Rehabil Med 2005;29:43-9.

9. Rosenbek JC, Robbins JA, Roecker EB, Coyle JL, Wood JL. A penetration-aspiration scale. Dysphagia 1996; 
11:93-8.

10. Kim JY, Koh ES, Kim HR, Chun SM, Lee SU, Jung SH. The diagnostic usefulness of the fiberoptic endoscopic evaluation of swallowing. J Korean Acad Rehabil Med 2011;35:14-22.

11. Wang TG, Wu MC, Chang YC, Hsiao TY, Lien IN. The effect of nasogastric tubes on swallowing function in persons with dysphagia following stroke. Arch Phys Med Rehabil 2006;87:1270-3.

12. Cook IJ, Dodds WJ, Dantas RO, Kern MK, Massey BT, Shaker R, et al. Timing of videofluoroscopic, manomet- ric events, and bolus transit during the oral and pharyngeal phases of swallowing. Dysphagia 1989;4:8-15.

13. Perlman AL, Palmer PM, McCulloch TM, Vandaele DJ. Electromyographic activity from human laryngeal, pharyngeal, and submental muscles during swallowing. J Appl Physiol 1999;86:1663-9.

14. Gomes CA Jr, Lustosa SA, Matos D, Andriolo RB, Waisberg DR, Waisberg J. Percutaneous endoscopic gastrostomy versus nasogastric tube feeding for adults with swallowing disturbances. Cochrane Database Syst Rev 2012;3:CD008096. 
Appendix 1. The functional dysphagia scale (FDS)

\begin{tabular}{|c|c|}
\hline & No. \\
\hline \multicolumn{2}{|l|}{ Oral phase } \\
\hline \multicolumn{2}{|l|}{ Lip closure } \\
\hline Intact & 0 \\
\hline Inadequate & 5 \\
\hline None & 10 \\
\hline \multicolumn{2}{|c|}{ Bolus formation } \\
\hline Intact & 0 \\
\hline Inadequate & 3 \\
\hline None & 6 \\
\hline \multicolumn{2}{|c|}{ Residue in oral cavity } \\
\hline None & 0 \\
\hline$<10$ & 2 \\
\hline $10-50$ & 4 \\
\hline$>50$ & 6 \\
\hline \multicolumn{2}{|c|}{ Oral transit time (s) } \\
\hline$\leq 1.5$ & 0 \\
\hline$>1.5$ & 6 \\
\hline \multicolumn{2}{|c|}{ Pharyngeal phase } \\
\hline \multicolumn{2}{|c|}{ Triggering of pharyngeal swallow } \\
\hline Normal & 0 \\
\hline Reduced & 10 \\
\hline \multicolumn{2}{|c|}{ Laryngeal elevation } \\
\hline Normal & 0 \\
\hline Reduced & 12 \\
\hline \multicolumn{2}{|c|}{ Nasal penetration } \\
\hline None & 0 \\
\hline$<10$ & 4 \\
\hline $10-50$ & 8 \\
\hline$>50$ & 12 \\
\hline \multicolumn{2}{|c|}{ Residue in valleculae (\%) } \\
\hline None & 0 \\
\hline$<10$ & 4 \\
\hline $10-50$ & 8 \\
\hline$>50$ & 12 \\
\hline \multicolumn{2}{|c|}{ Residue in pyriform sinuses (\%) } \\
\hline None & 0 \\
\hline$<10$ & 4 \\
\hline $10-50$ & 8 \\
\hline$>50$ & 12 \\
\hline \multicolumn{2}{|c|}{ Coating of pharyngeal wall after swallow } \\
\hline No & 0 \\
\hline Yes & 10 \\
\hline \multicolumn{2}{|c|}{ Pharyngeal transit time (s) } \\
\hline$\leq 1.0$ & 0 \\
\hline$>1.0$ & 4 \\
\hline Total score & 100 \\
\hline
\end{tabular}

Appendix 2. The modified penetration-aspiration scale (mPAS)

\begin{tabular}{cc}
\hline Scale & \\
\hline 1 & Material does not enter the airway \\
2 & $\begin{array}{c}\text { Material enters the airway, remains above } \\
\text { the vocal folds }\end{array}$ \\
3 & $\begin{array}{c}\text { Material enters the airway, contacts the } \\
\text { vocal folds }\end{array}$ \\
4 & $\begin{array}{c}\text { Material enters the airway, passes below } \\
\text { the vocal folds and effort is made to eject }\end{array}$ \\
5 & $\begin{array}{c}\text { Material enters the airway, passes below } \\
\text { the vocal folds and no effort is made to eject }\end{array}$ \\
\hline
\end{tabular}

